\title{
Regularizing QCD with staggered fermions and the fourth root trick
}

\author{
Claude Bernard \\ Department of Physics, Washington University, Saint Louis, MO 63130, USA \\ E-mail: cb@lump.wustl.edu
}

\section{Maarten Golterman}

Department of Physics and Astronomy, San Francisco State University,

San Francisco, CA 94132, USA

E-mail: maartenestars.sfsu.edu

\section{Yigal Shamir}

School of Physics and Astronomy, Raymond and Beverly Sackler Faculty of Exact Sciences,

Tel-Aviv University, Ramat Aviv, 69978, Israel

E-mail: shamirepost.tau.ac.il

\begin{abstract}
We investigate the properties of staggered-fermion lattice QCD in which the fourth root of the fermion determinant is taken. We show that this theory is non-local at non-zero lattice spacing $a$, and that the non-locality is caused by the breaking of taste symmetry at $a \neq 0$. We then present a renormalization-group based argument that the theory restores taste symmetry in the continuum limit. As a consequence the theory is local in that limit, and falls into the correct universality class. Finally, we argue that the correct effective theory for the physics of Goldstone bosons at $a \neq 0$ is given by staggered chiral perturbation theory with the replica trick.
\end{abstract}




\section{Introduction and overview}

Staggered fermions [1] are commonly used for numerical computations in lattice QCD, in which it is now becoming standard to include the fermion determinants for all three light quark flavors, up, down, and strange. They have an exact chiral $U(1)$ symmetry at zero quark mass [2], implying that the critical value of the bare quark mass for each quark flavor is known to be zero. Furthermore, staggered fermions are numerically inexpensive, and the combination of these two facts has made it possible to reach the chiral regime of light pseudo-scalar Goldstone boson (GB) masses essential for phenomenology.

In practice, a separate staggered fermion field is introduced for each physical flavor, with a single-site mass term to describe their masses $m_{u}, m_{d}$ and $m_{s}$. However, each staggered fermion describes four degenerate quarks in the continuum limit, commonly referred to as "tastes," and one ends up with a theory with four up, four down, and four strange quarks, with (for non-degenerate quark masses) a $U(4)_{u} \times U(4)_{d} \times U(4)_{s}$ vector-like symmetry. In order to remedy this problem, one starts from the observation that if indeed each staggered fermion field describes four degenerate tastes in the limit of vanishing lattice spacing $a$, one would expect that the determinant of the staggered Dirac operator $D_{\text {stag }}$ factorizes as

$$
\operatorname{Det}\left(D_{\text {stag }}(m)\right) \sim \operatorname{Det}^{4}\left(D_{\text {continuит }}(m)\right), \quad a \rightarrow 0,
$$

where $m$ is the quark mass. The idea is then to take the fourth root, $\operatorname{Det}^{1 / 4}\left(D_{\text {stag }}\right)$ for each flavor in the generation of the gauge-field ensemble on which observables are computed. At the diagrammatic level, this multiplies each sea-quark loop by $1 / 4$, thus correcting for the too-many tastes per quark flavor present in the theory without the fourth root. Since the staggered determinant is positive for any $m \neq 0$, and the continuum determinant is (formally) positive for any $m>0$, one picks the positive fourth root. ${ }^{1}$ Since the continuum determinant is only positive for $m>0$, the trick only works for positive physical quark mass (see, however, Refs. [3, 4]).

Obviously, the argument for this trick is heuristic. Factorization as in Eq. (1.1) can never be exact: first, the right-hand side is not well-defined, and second, the ultraviolet (UV) eigenvalues of $D_{\text {stag }}$ will never form taste quartets. But we expect that quartets of eigenvalues will form at physical scales for small enough lattice spacing, while UV effects can be absorbed into a renormalization of the gauge coupling and masses, and thus that factorization will effectively take place in the continuum limit, validating the use of the fourth-root trick. We note that this expectation is based on the generally-assumed properties of the continuum limit of unrooted staggered fermions (to which Eq. (1.1) refers), about which there is little doubt. This observation plays a key role in establishing the validity of regulating QCD with rooted staggered fermions [5].

We thus face the following set of questions, all of which need to be answered to establish the validity of working with rooted staggered fermions:

1. The prescription for regulating QCD with staggered fermions and the fourth-root trick is unambiguous and easy to implement. The question is whether this prescription is a regulator like any other or not. We will argue in Sec. 2 that it is not, in the sense that the theory is non-local at $a \neq 0[6]$.

\footnotetext{
${ }^{1}$ A negative staggered quark mass can be made positive through a $U(1)_{\varepsilon}$ rotation.
} 
2. Given this result, the next question is whether the continuum limit can be taken, and whether that limit is in the correct universality class. In Sec. 3 we discuss a renormalization-group (RG) framework through which to address this question [5]. To motivate the use of the RG, we note that it deals with two issues already mentioned: it separates UV scales from physical scales, and it makes it possible to define a $U(4)$ taste-invariant theory at some fixed coarse lattice spacing $a_{c} \ll \Lambda_{Q C D}^{-1}$, obtained by blocking the original staggered theory on a fine lattice with spacing $a=a_{f}$ an infinite number of times (so that $a_{f} / a_{c} \rightarrow 0$ ). In other words, it helps in defining the right-hand side of Eq. (1.1). Our arguments give strong evidence that indeed the non-locality vanishes in the continuum limit and the correct universality class is obtained using the fourth-root trick, i.e., that it is a valid regulator. All the key elements of the argument can be confirmed by perturbative calculations and/or concrete numerical tests.

3. In view of the answers to questions 1 and 2, a third issue arises. Lattice computations are performed at non-zero $a$, and they are thus affected by the non-local nature of the regulator. Even though the answer to the second question implies that these unphysical effects go away in the continuum limit, one needs to understand them in order to analyze and fit the data generated at $a \neq 0$. Here, this issue is addressed for the physics of GBs. In Sec. 4 we argue that the correct effective theory describing GBs is given by staggered chiral perturbation theory (S $\chi \mathrm{PT}$ ) plus the replica trick [7]. At a pedestrian level, the replica trick refers to the fact that sea-quark loops are corrected by a factor of four "by hand," after identifying the quark-flow diagrams underlying a S $\chi \mathrm{PT}$ calculation. The replica trick thus implements the fourth root at the level of the effective theory.

Before embarking on the topics outlined above, let us first consider the continuum limit in more detail. If the continuum limit exists and is in the correct universality class, it can be (formally) described by the path integral (including sources for mesons)

$$
Z_{\text {continuиm }}(J)=\int \mathscr{D} \mathscr{U} e^{-S_{g}(\mathscr{U})} \operatorname{Det}^{1 / 4}((D(\mathscr{U})+M) \otimes \mathbf{1}+J),
$$

where $S_{g}(\mathscr{U})$ is the gauge action. The Dirac operator $D(\mathscr{U})$ carries no taste index, and $\mathbf{1}$ is the $4 \times 4$ identity matrix acting on the taste index. The mass matrix is $M=\operatorname{diag}\left(m_{u}, m_{d}, m_{s}\right)$, with all masses positive. The source $J$ is a matrix in spin, flavor and taste space. If we project the complete set of correlation functions generated by this partition function onto the taste-singlet set by setting $J=\tilde{J} \otimes \mathbf{1}$, the fourth root reduces to $\operatorname{Det}((D(\mathscr{U})+M)+\tilde{J})$, and it becomes obvious that the taste-singlet sector is the physical sector, containing precisely the correlation functions for unquenched three-flavor QCD. This simple observation implies that no paradoxes based on symmetry arguments ${ }^{2}$ can arise [3].

Of course, many unphysical correlation functions exist in the theory defined by Eq. (1.2), and they can be generated by using a source $J$ that is not proportional to the identity in taste space. However, because of the exact $S U(4)_{\text {taste }}$ vector symmetry of the theory, flavor non-singlet but taste-singlet operators can be related to taste non-singlet ones, for example,

$$
\bar{u} \gamma_{5} d \underset{\text { SU(4)taste }}{\longrightarrow} \bar{u} \gamma_{5} \Xi d
$$

\footnotetext{
${ }^{2}$ as presented for instance in Ref. [8]
} 
where $\Xi$ is an $S U(4)_{\text {taste }}$ rotation acting (for example) on the down quark. If we choose $\Xi=\xi_{5}$, the operator on the right-hand side corresponds to the exact GB related to $U(1)_{\varepsilon}$ symmetry. This is the pion field usually used in simulations. Equation (1.3) tells us that this is equivalent to using the physical operator $\bar{u} \gamma_{5} d$. However, if one is interested in flavor-singlet physics, in which mixing with gluonic states can occur, one must use taste-singlet operators, in accord with the fact that gluons do not have taste. Indeed, the trick of Eq. (1.3) will not work if both the quark and the anti-quark have the same flavor. In cases with exact flavor symmetry, it may nevertheless be possible to rotate a flavor-neutral interpolating field first into a flavor-charged one, and then make taste rotations as in Eq. (1.3). For example, if $m_{u}=m_{d}$, we can rotate a taste-singlet $\pi^{0}$ field into a taste-singlet $\pi^{+}$ field and then use Eq. (1.3) to relate it to a taste-nonsinglet $\pi^{+}$field. However, this will not work if $m_{u} \neq m_{d}$, when a $\pi^{0}$ can mix with gluonic states.

\section{Non-locality at $a \neq 0$}

It is straightforward to establish the non-locality of the rooted theory at non-zero lattice spacing. We proceed by assuming the theory to be local, and derive a contradiction [6]. Assume that a local Dirac operator $D$ exists such that (at $a \neq 0$ )

$$
\operatorname{Det}^{1 / 4}\left(D_{\text {stag }}\right)=\operatorname{Det}(D) \exp \left(-\delta S_{\text {eff }} / 4\right),
$$

with $\delta S_{\text {eff }}$ a local functional of the gauge field which does not contribute to any long-distance effects (apart from a possible renormalization of the gauge coupling). Take the fourth power:

$$
\operatorname{Det}\left(D_{\text {stag }}\right)=\operatorname{Det}\left(D_{4 t}\right) \exp \left(-\delta S_{\text {eff }}\right), \quad D_{4 t}=D \otimes \mathbf{1} .
$$

The operator $D_{4 t}$ describes a theory with an exact $U(4)$ taste symmetry. We may now compare the spectra of hadron multiplets in the two theories at non-zero lattice spacing, considering for instance the pions. The theory defined by $D_{4 t}$ has fifteen degenerate pions ${ }^{3}$ in the adjoint representation of $S U(4)$. But the theory described by $D_{\text {stag }}$ is well known to have a spectrum of fifteen nondegenerate pions (with only one "exact" pion corresponding to $U(1)_{\varepsilon}$ symmetry), due to the fact that the staggered symmetry group is much smaller than $S U(4)$ [9]. It follows that, contrary to our assumption, $\delta S_{\text {eff }}$ has to know about long distance effects! Thus the staggered theory with the fourth root is non-local.

While this concludes our basic argument, it is instructive to discuss this result in more detail. In order to do this, we first go to the so-called taste basis, by defining [10]

$$
D_{\text {taste }}^{-1}=\alpha^{-1}+Q D_{\text {stag }}^{-1} Q^{\dagger},
$$

where $\alpha$ is a constant of order $1 / a$, and $Q$ is a (gauge-covariant) unitary matrix connecting the one-component and taste bases [11]. The contact term $\alpha^{-1}$ is new, but it has no effect on the longdistance physics. Choosing $\alpha$ finite (instead of infinite, which would turn Eq. (2.3) into the usual basis transformation of Ref. [11]) is advantageous for setting up the RG framework in the next section, as will become clear below. With $0<\alpha<\infty$, we have that

$$
\begin{aligned}
\operatorname{Det}\left(D_{\text {stag }}\right) & =\operatorname{Det}\left((\alpha G)^{-1}\right) \operatorname{Det}\left(D_{\text {taste }}\right), \\
(\alpha G)^{-1} & =\frac{1}{\alpha} D_{\text {stag }}+Q^{\dagger} Q=\frac{1}{\alpha} D_{\text {stag }}+1 .
\end{aligned}
$$

\footnotetext{
${ }^{3}$ Here we will use the word "pion" for any meson that becomes an exact GB in the continuum and chiral limits.
} 
For $\alpha \sim 1 / a$, the effective action defined by $\left.\log \operatorname{Det}\left((\alpha G)^{-1}\right)\right)$ is local, because $(\alpha G)^{-1}$ is a lattice Dirac operator with a bare mass of order $1 / a$. This effective action vanishes as $\alpha \rightarrow \infty$.

We now may split $D_{\text {taste }}$ into taste-invariant and non-invariant parts:

$$
D_{\text {taste }}=D_{1} \otimes \mathbf{1}+\sum_{A} D_{A} \otimes \Xi_{A}
$$

where the $\Xi_{A}$ are fifteen traceless hermitian matrices spanning the taste space. We thus have that

$$
\log \operatorname{Det}\left(D_{\text {taste }}\right)=4 \log \operatorname{Det}\left(D_{1}\right)+\log \operatorname{Det}\left(1+D_{1}^{-1} \sum_{A} D_{A} \otimes \Xi_{A}\right)
$$

Both $D_{1}$ and the $D_{A}$ are local, but the effective action defined by the last term on the right-hand side is not. On the other hand, the theories defined by $\log \operatorname{Det}\left(D_{\text {taste }}\right)$ or $\log \operatorname{Det}\left(D_{1}\right)$ separately are obviously local. ${ }^{4}$ This makes it clear where the non-locality of the rooted theory comes from: it originates directly from the taste breaking present in the unrooted theory. But it also makes it clear how it may go away in the continuum limit: the $D_{A}$ are of order $a$, and thus constitute a set of irrelevant operators. If no quantum effects change this observation, taste symmetry should be restored in the continuum limit, and, mutatis mutandis (as we will argue in the next section), the rooted theory should become local. Equation (2.6) is an example of Eq. (2.2) with $D=D_{1}$, but with $\delta S_{\text {eff }}$ non-local.

Working out $D_{\text {taste }}$ in the free theory in momentum space, we find that

$$
D_{\text {taste }}=\frac{\sum_{\mu} i\left[\gamma_{\mu} \otimes \mathbf{1}\right] \bar{p}_{\mu}+m+\frac{1}{\alpha}\left(\hat{p}^{2}+m^{2}\right)+\frac{1}{2} \sum_{\mu}\left[\gamma_{5} \otimes \xi_{\mu} \xi_{5}\right] \hat{p}_{\mu}^{2}}{1+\frac{2 m}{\alpha}+\frac{1}{\alpha^{2}}\left(\hat{p}^{2}+m^{2}\right)}
$$

in which $\bar{p}_{\mu} \equiv \sin p_{\mu}, \hat{p}_{\mu}=2 \sin \left(p_{\mu} / 2\right)$, and $\hat{p}^{2} \equiv \sum_{\mu} \hat{p}_{\mu}^{2}$. The last term in the numerator of Eq. (2.7) is what removes the fermion doublers in the usual taste-basis action [11] (i.e., for $\alpha=\infty$ ), at the price of breaking the taste symmetry explicitly. The taste-invariant Dirac operator $D_{1}$ in Eq. (2.5) is constructed by dropping that term. An important observation is that $D_{1}$ also has no doublers, because of the Wilson-like term proportional to $1 / \alpha \sim a$ in the numerator. This is a prerequisite for having a taste-invariant theory in the same universality class as the staggered theory, making feasible the comparison of pairs of such theories in Sec. 3.

At this point, it is useful to reflect on the nature of the non-locality in the case at hand. ${ }^{5}$ In general, the tendency is to "stay away" from non-local field theories, whereas here we argue that in this case there is no need to: the continuum limit is in the desired universality class ( $c f$. next section), and the non-local behavior at $a \neq 0$ can be understood in detail ( $c f$. Sec. 4). In the theory with the fourth root, there are "too many" pions. While we should end up with eight pions (in the theory with three flavors), before taking the fourth root the theory has many more, because of the unphysical, extra taste degree of freedom. Of course, taking the fourth root is precisely intended to remove the surplus of pions, by inserting the appropriate number of factors $1 / 4$ into various terms making up the correlation functions describing the propagation of the pions. If all pions are members of exact taste multiplets, this should work, as it would in the theory described by

\footnotetext{
${ }^{4}$ Note that when we say an effective action is non-local, we mean that it cannot be written as a fermion path integral with any local action.

${ }^{5}$ See also Ref. [12].
} 
Eq. (1.2). The problem is that at non-zero lattice spacing taste symmetry is broken, so that within one taste multiplet, the pion masses are (to leading order in $\mathrm{S} \chi \mathrm{PT}$ ) given by

$$
\left(m_{\pi}^{A}\right)^{2}=\left(m_{\pi}^{G B}\right)^{2}+c^{A} a^{2} \Lambda_{Q C D}^{4}
$$

in which GB denotes the one exact Goldstone boson, and the $c^{A}$ are numerical coefficients which do not vanish except when the taste index $A$ refers to the exact GB. This "mismatch" between masses shows up as violations of unitarity, a manifestation of the non-locality of rooted staggered fermions at non-zero lattice spacing. For explicit examples, see Refs. [13, 14, 7]. We note that these arguments extend to other hadron multiplets as well; we just focused on the pion sector because it contains the lightest excitations in the theory.

One way to rephrase this observation is by noting that there are two independent infrared (IR) scales in the theory, one being the physical pion mass $m_{\pi}^{G B}$ governed by the quark mass, and the other $a \Lambda_{Q C D}^{2}$, which is generated by the taste splitting of low-lying eigenvalues of the staggered Dirac operator. In other words, there are two different scales which control the IR behavior of the theory, a physical one and an unphysical one. As is often the case, the order in which IR scales are taken to zero matters, and in this case it is clear that the right order is to first take the unphysical scale $a \Lambda_{Q C D}^{2}$ to zero if one wishes to study the chiral limit [15,3]. Of course, taking $a \Lambda_{Q C D}^{2}$ to zero is done by taking the continuum limit. In addition, it is clear that, in view of the unitarity violations at $a \neq 0$, one should also take the continuum limit before continuing the theory to Minkowski space.

\section{Renormalization-group analysis of (rooted) staggered fermions}

The (thus far formal) argument based on Eq. (1.2) says that rooted staggered fermions provide a valid regularization of QCD if exact taste symmetry is recovered in the continuum limit. We will now build an adequate non-perturbative framework where well-defined statements can be made about the continuum limit. Using this framework we will argue that under plausible, and testable, assumptions, rooted staggered fermions indeed provide a valid regularization of QCD.

\subsection{Strategy}

In Sec. 2 we discussed how taste-splittings manifest themselves in physical observables. We now take a step back and examine taste-symmetry violations at the most fundamental level: in the spectrum of the staggered Dirac operator. On gauge-field configurations drawn from a (rooted or unrooted) dynamical ensemble, what one expects to find is that the low-lying eigenvalues arrange themselves nicely into almost-degenerate taste quartets $[16,17]$. But for larger eigenvalues the taste symmetry deteriorates until, finally, at the cutoff, taste degeneracy is completely lost.

If taste symmetry must be lost at the cutoff scale, let's get rid of all cutoff-scale physics. The way to eliminate cutoff-scale effects is to apply renormalization-group (RG) block transformations. With each blocking step the lattice spacing is doubled, until an effective theory on a coarse lattice with spacing $a_{c}$ is reached. As we approach the continuum limit we fix the QCD scale $\Lambda_{Q C D}$ and the renormalized quark masses by adjusting the bare parameters. We fix the coarse-lattice spacing $a_{c}$ too: each time we make an additional blocking step, we simultaneously decrease the fine-lattice spacing $a_{f}$ by a factor of two. The limit of infinitely many blocking steps implies the usual continuum limit, $a_{f} \rightarrow 0$. The coarse-lattice spacing provides a new, intermediate distance 
scale, $a_{f} \ll a_{c} \ll \Lambda_{Q C D}^{-1}$. RG-blocking eliminates from the theory all the fermion modes with eigenvalues (well) above the coarse-lattice cutoff $1 / a_{c}$. The remaining eigenmodes, those of the blocked Dirac operator, all have eigenvalues that become vanishingly small in units of the underlying cutoff $1 / a_{f}$. Because $a_{c}$ is kept fixed, we expect that all these eigenvalues will uniformly show the quartet structure required by taste symmetry. In other words, the blocked staggered Dirac operator will acquire the taste-diagonal form of Eq. (1.2) in the continuum limit, and its fourth root will correspond to a local one-taste theory.

There are several reasons for choosing $a_{c} \ll \Lambda_{Q C D}^{-1}$. First, we take as criterion for the theory in the continuum limit to be local the requirement that the coarse-lattice action be local on the scale $a_{c}$. This only makes sense if $a_{c} \ll \Lambda_{Q C D}^{-1}$. We also want the complete set of coarse-lattice observables to be rich enough to extract all of the QCD physics. Again this requires that $1 / a_{c}$ will be a high-energy scale relative to the QCD scale. A third reason will be encountered below.

The blocking framework constructed below is designed to make the most out of our understanding of uncontroversial lattice regularizations of QCD and, in particular, of unrooted staggered fermions. A fairly standard blocking framework is set up for ordinary (unrooted) staggered fermions in a form where the adaptations required for rooted staggered fermions are minimal.

The novel element is the introduction of comparison blocked-lattice theories with exact taste invariance. At each blocking level, a new theory with exact taste symmetry is constructed by simply dropping the taste-breaking part of the blocked Dirac operator. This is equivalent to dropping the rightmost, non-local term of Eq. (2.6), except that it is done after a blocking process. In the rooted theory, the result will be that each of the so-obtained reweighted theories is a local one-taste theory. Of course, while all by itself RG-blocking leaves the physics invariant, the reweighted theories constructed at different blocking levels are different from one another, as well as from the original (rooted or not) staggered theory.

Taste-breaking effects that survive blocking become smaller and smaller with each additional blocking step. As a result, at each blocking level, the difference between a (blocked) staggered theory and the corresponding reweighted theory gets smaller. In the (continuum-)limit of infinitely many blocking steps, the difference vanishes for every observable. ${ }^{6}$ Because each reweighted theory is local and belongs to the correct universality class, the same applies to rooted staggered fermions in the continuum limit.

Clearly, this amounts to a set of highly non-trivial claims which require a detailed justification. Several key steps of the argument basically work in the same way for the unrooted and the rooted staggered theories. Note that for the ordinary (unrooted) theory, they lead to the uncontroversial conclusion that the continuum-limit theory consists of four degenerate quark species per staggered field. The last crucial step of the argument is, however, more complicated in the rooted theory.

\subsection{The RG blocking framework}

We first introduce our notation. We will perform $n+1$ blocking steps labeled $k=0, \ldots, n$. The $k^{\text {th }}$ lattice spacing is $a_{k}=2^{k+1} a_{f}$ where as already mentioned $a_{f}$ is the fine-lattice spacing and $a_{c} \equiv a_{n}$ is the coarse-lattice spacing. The $k=0$ step, already discussed in Sec. 2 , is special. It transforms the staggered field from its usual one-component basis to a taste basis, which is then

\footnotetext{
${ }^{6}$ It is necessary to assume that $m \neq 0$ for all flavors.
} 
retained in all subsequent blocking steps. Thinning out of the fermion and gauge-field degrees of freedom occurs at each step, except for the $k=0$ step where the fermions are not thinned out but roughly speaking only undergo a change of basis. In order to avoid overly cluttered notation we will consider a singe-flavor theory in this section. The generalization is trivial.

We first set up the blocking framework for the ordinary (unrooted) staggered theory. ${ }^{7}$ Blocking in the rooted theory will be introduced later. The unrooted partition function is

$$
Z=\int \mathscr{D} \mathscr{U} \mathscr{D} \chi \mathscr{D} \bar{\chi} \exp \left(-S_{g}-\bar{\chi} D_{\text {stag }} \chi\right)
$$

where $\chi(x), \bar{\chi}(x)$ is a one-flavor staggered field, and $U_{\mu, x}$ denotes the link variable. The gauge field as a whole will be denoted $\mathscr{U}=\left\{U_{\mu, x}\right\}$. Again $S_{g}=S_{g}(\mathscr{U})$ is the gauge action and $D_{\text {stag }}=$ $D_{\text {stag }}(\mathscr{U})$ is the staggered Dirac operator.

The coordinates of the $k^{\text {th }}$ step blocked lattice will be denoted $\tilde{x}^{(k)}$. The fermion and antifermion fields on that lattice are $\psi_{\alpha i}^{(k)}\left(\tilde{x}^{(k)}\right)$ and $\bar{\psi}_{\alpha i}^{(k)}\left(\tilde{x}^{(k)}\right)$ respectively. The indices $\alpha$ and $i$, both ranging from one to four, are the Dirac and the taste index respectively. The blocked link variables will be denoted $V_{\mu, \tilde{x}^{(k)}}^{(k)}$, and the blocked gauge field as a whole $\mathscr{V}^{(k)}=\left\{V_{\mu, \tilde{x}^{(k)}}^{(k)}\right\}$.

RG-blocking is performed by multiplying the integrand of the path integral by one, written in a sophisticated form, and then interchanging the order of integrations. Fermion blocking is always done with a gaussian kernel that, for $k \geq 1$, takes the explicit form

$$
1=\alpha_{k}^{-16 N_{k}} \int \mathscr{D} \psi^{(k)} \mathscr{D} \bar{\psi}^{(k)} \exp \left[\alpha_{k}\left(\bar{\psi}^{(k)}-\bar{\psi}^{(k-1)} Q^{(k) \dagger}\right)\left(\psi^{(k)}-Q^{(k)} \psi^{(k-1)}\right)\right] .
$$

Here $\alpha_{k}$ is a blocking parameter of mass dimension one, that is naturally taken to be $O\left(a_{k}^{-1}\right)$, and $N_{k}$ is the number of sites of the $k^{\text {th }}$ lattice. The blocking kernel $Q^{(k)}=Q^{(k)}\left(\mathscr{V}^{(k-1)}\right)$ is ultra-local and gauge covariant. It defines a linear mapping from the sites of a $2^{4}$ hypercube on the $(k-1)^{\text {th }}$ lattice to the corresponding single site of the $k^{\text {th }}$ lattice. Apart from the $k=0$ step (Sec. 2), $Q^{(k)}$ acts trivially on the Dirac and taste indices. For the gauge field we assume a conventional ultra-local blocking kernel whose details are not needed.

We begin by introducing the kernels for the $n+1$ blocking steps. However, we do not integrate over any gauge field yet, for a reason that will become clear shortly. We do integrate over all the fermion fields except those living on the coarse lattice. This can be done in closed form because the fermion integrals are gaussian. The result is

$$
\begin{array}{rl}
Z=\int \mathscr{D} & \mathscr{U} \\
& \mathscr{D} \mathscr{V}^{(0)} \mathscr{D} \mathscr{V}^{(1)} \ldots \mathscr{D} \mathscr{V}^{(n)} \exp \left(-S_{g}-\sum_{k=0}^{n} \mathscr{K}_{B}^{(k)}-\sum_{k=0}^{n} S_{\text {eff }}^{(k)}\right) \\
\times \int d \psi^{(n)} d \bar{\psi}^{(n)} \exp \left(-\bar{\psi}^{(n)} D_{n} \psi^{(n)}\right)
\end{array}
$$

Here $\mathscr{K}_{B}^{(0)}=\mathscr{K}_{B}^{(0)}\left(\mathscr{V}^{(0)}, \mathscr{U}\right)$ and $\mathscr{K}_{B}^{(k)}=\mathscr{K}_{B}^{(k)}\left(\mathscr{V}^{(k)}, \mathscr{V}^{(k-1)}\right), 1 \leq k \leq n$, are the gauge-field blocking kernels. The UV fermion modes that have been integrated out at the $k^{\text {th }}$ step give rise to the effective action

$$
S_{e f f}^{(k)}=\log \operatorname{det}\left(G_{k}\right)
$$

\footnotetext{
${ }^{7}$ We assume a fixed, finite physical volume. By assumption all quarks are massive, and we expect no subtlety when taking the thermodynamical limit.
} 
The operators $D_{k}$ and $G_{k}^{-1}$ may be constructed iteratively (for the $k=0$ step see Sec. 2)

$$
\begin{aligned}
D_{k} & =\alpha_{k}-\alpha_{k}^{2} Q^{(k)} G_{k} Q^{(k) \dagger}, \\
G_{k}^{-1} & =D_{k-1}+\alpha_{k} Q^{(k) \dagger} Q^{(k)} .
\end{aligned}
$$

Let us briefly summarize the important properties of these operators in the free theory [10]. The operator $D_{k}$ is gap-less for $m=0$, with its low-lying eigenmodes coming from $p \sim 0$. In Eq. (3.6) the addition of the blocking-kernel term lifts the small eigenvalues and creates an $O\left(\alpha_{k}\right)=$ $O\left(1 / a_{k}\right)$ gap in the spectrum of $G_{k}^{-1}$. One can then prove iteratively that $D_{k}, G_{k}^{-1}$, and $G_{k}$ are all local operators [18]. By this we mean that their matrix elements connecting sites $\tilde{x}^{(k)}$ and $\tilde{y}^{(k)}$ vanish exponentially with the separation, with an $O\left(a_{k}\right)$ decay length.

Expanding the free, coarse-lattice Dirac operator $D_{n}$ to second order in $p$ and $m$ gives

$$
D_{n}(p)=m+i[\not p \otimes \mathbf{1}]+a_{f} \sum_{\mu}\left[\gamma_{5} \otimes \xi_{5} \xi_{\mu}\right] p_{\mu}^{2}-R_{n}(m+i[\not p \otimes \mathbf{1}])^{2}+\cdots .
$$

where $R_{n}=\sum_{k=0}^{n}(16)^{n-k} / \alpha_{k}$ is $O\left(a_{c}\right)$. The shown taste-breaking term originates from the last term in the numerator of Eq. (2.7). Because $|p| \lesssim a_{c}^{-1}$, this irrelevant term is $O\left(a_{f} / a_{c}^{2}\right)$, which is just the scaling required by its engineering dimension. In the limit $n \rightarrow \infty$ the blocked Dirac operator becomes taste-diagonal. For $m=0$, the operator $D_{n}$ satisfies a Ginsparg-Wilson (GW) relation. Thus, through RG blocking, the taste-violating mechanism for removing the doublers is gradually taken over by the taste-conserving and chiral symmetry conserving GW mechanism.

In both the free and the interacting theories the inverse blocked Dirac operator satisfies ${ }^{8}$

$$
D_{k}^{-1}=\alpha_{k}^{-1}+Q^{(k)} D_{k-1}^{-1} Q^{(k) \dagger} .
$$

Using Eq. (3.8) recursively we may express the coarse-lattice fermion propagator as the fine-lattice propagator between smeared sources defined by the product of the blocking kernels. ${ }^{9}$ Equation (3.8) is in fact a special case of a completely general pattern. Considering any operator $\mathscr{O}^{(c)}$ constructed from the coarse-lattice fields, we may "undo" the blocking by performing the integration over all the blocked (gauge-field and fermion) fields we have introduced, resulting in an operator $\mathscr{O}^{(f)}$ that depends only on the fine-lattice fields. This operation defines a pull-back mapping $\mathscr{T}^{(f, c)}: \mathscr{O}^{(c)} \rightarrow \mathscr{O}^{(f)}$. The pull-back mapping is ultra-local (because the blocking kernels are) and it preserves expectation values: $\left\langle\mathscr{T}^{(f, c)} \mathscr{O}^{(c)}\right\rangle_{f}=\left\langle\mathscr{O}^{(c)}\right\rangle_{c}$.

The existence of the pull-back mapping means that every coarse-lattice observable is at the same time also a fine-lattice observable. This "kinematical" feature has an important dynamical consequence: The coarse-lattice observable must preserve the constraints that follow from all the fine-lattice symmetries, even if (as it actually happens) some of these symmetries are not manifestly preserved by the blocking (for more details, see Ref. [5]).

We next introduce a family of comparison theories as follows. First, as in Eq. (2.5) we split the blocked Dirac operator into its taste-invariant and taste-breaking parts

$$
D_{n}=\widetilde{D}_{n} \otimes \mathbf{1}+\Delta_{n},
$$

\footnotetext{
${ }^{8}$ For the $k=0$ step, Eq. (3.8) reduces to Eq. (2.3).

${ }^{9}$ This is true up to the contact term in the propagator, which vanishes for $\tilde{x}^{(k)} \neq \tilde{y}^{(k)}$.
} 
where $\widetilde{D}_{n}$ carries no taste index, and $\Delta_{n}$ is traceless on the taste index. The replacement $\Delta_{n} \rightarrow t \Delta_{n}$, where $0 \leq t \leq 1$, then allows us to gradually "turn off" all the taste breaking. For $t=0$, exact taste symmetry is restored by hand. Performing this at the level of the (blocked) partition function generates a family of interpolating theories

$$
\begin{array}{rl}
Z_{n}^{\text {inter }}(t)=\int \mathscr{D} \mathscr{U} & \mathscr{D} \mathscr{V}^{(0)} \mathscr{D} \mathscr{V}^{(1)} \ldots \mathscr{D} \mathscr{V}^{(n)} \exp \left(-S_{g}-\sum_{k=0}^{n} \mathscr{K}_{B}^{(k)}-\sum_{k=0}^{n} S_{e f f}^{(k)}\right) \\
\times \int d \psi^{(n)} d \overline{\boldsymbol{\psi}}^{(n)} \exp \left[-\overline{\boldsymbol{\psi}}^{(n)}\left(\widetilde{D}_{n} \otimes \mathbf{1}+t \Delta_{n}\right) \psi^{(n)}\right] .
\end{array}
$$

In the next subsection we will argue that these interpolating theories are all local. They clearly belong to the same universality class as the original staggered theory.

We now come to the rooted staggered theory defined by

$$
Z^{\text {root }}=\int \mathscr{D} \mathscr{U} \exp \left(-S_{g}\right) \operatorname{Det}^{1 / 4}\left(D_{\text {stag }}\right) .
$$

The gaussian fermion-blocking transformations readily lead to identities for the fermion determinant that hold for any given set of values of the (original and blocked) gauge fields. This allows us to write down the analogue of Eq. (3.3) for the rooted theory

$$
Z^{r o o t}=\int \mathscr{D} \mathscr{U} \mathscr{D}^{(0)} \mathscr{D} \mathscr{V}^{(1)} \ldots \mathscr{D} \mathscr{V}^{(n)} \exp \left(-S_{g}-\sum_{k=0}^{n} \mathscr{K}_{B}^{(k)}-\frac{1}{4} \sum_{k=0}^{n} S_{e f f}^{(k)}\right) \operatorname{Det}^{1 / 4}\left(D_{n}\right)
$$

Of course, as already noted in Eq. (2.6), after $n$-step blocking we still cannot express the rooted determinant as a local path integral. This is made possible only after dropping the taste-breaking part $\Delta_{n}$ completely. We then arrive at a reweighted theory

$$
\begin{array}{rl}
Z_{n}^{\text {reweigh }}=\int \mathscr{D} & \mathscr{U} \mathscr{D}^{(0)} \mathscr{D} \mathscr{V}^{(1)} \ldots \mathscr{D} \mathscr{V}^{(n)} \exp \left(-S_{g}-\sum_{k=0}^{n} \mathscr{K}_{B}^{(k)}-\frac{1}{4} \sum_{k=0}^{n} S_{\text {eff }}^{(k)}\right) \\
& \times \int d q^{(n)} d \bar{q}^{(n)} \exp \left(-\bar{q}^{(n)} \widetilde{D}_{n} q^{(n)}\right),
\end{array}
$$

where we used $\operatorname{Det}^{1 / 4}\left(\widetilde{D}_{n} \otimes \mathbf{1}\right)=\operatorname{Det}\left(\widetilde{D}_{n}\right)$. The coarse-lattice quark fields $q^{(n)}, \bar{q}^{(n)}$ carry no taste index. In the next subsection, we will argue that the reweighted theories $Z_{n}^{\text {reweigh }}$ are local too, and that they fall into the right universality class. (This puts on a solid basis the observations made below Eq. (1.2).) For rooted (unrooted) staggered fermions, we will show that each observable of the $n^{\text {th }}$ reweighted (interpolated) theory has the same $n \rightarrow \infty$ limit as the corresponding observable of the blocked staggered theory.

\subsection{Validity of rooted staggered fermions in the continuum limit}

Ordinary staggered fermions define a renormalizable lattice theory. The renormalizability of the ordinary staggered theory has not yet been established to all orders. Yet there is no real reason to doubt that all-orders renormalizability holds in the unrooted theory. As shown in Ref. [9], any relevant or marginal term not already present in the original staggered action is forbidden by its symmetries, and thus will not be induced by loop corrections.

Renormalizability readily generalizes to the rooted theory. This was first observed in Ref. [19]. For a recent detailed discussion, see Ref. [12]. Consider first a theory with $n_{R}$ copies, or replicas, 
of identical staggered fields. The counterterms at any order are polynomials in (the integer) $n_{R}$. Now, in perturbation theory, rooting amounts to multiplying each staggered-fermion loop by $1 / 4$. Therefore the same counterterms will suffice to renormalize the rooted theory when we substitute $n_{R} \rightarrow 1 / 4$. While locality is lost with rooting, renormalizability is saved. The theory remains tightly constrained by power counting and symmetries.

Our third motivation for choosing the coarse-lattice spacing to satisfy $a_{c} \ll \Lambda^{-1}$, is that QCD is weakly coupled at short distances. The existence of a weak-coupling regime depends on renormalizability, but it does not necessarily require locality. ${ }^{10}$ Any four-dimensional lattice gauge theory whose Boltzmann weight contains a fermion determinant raised to a real positive power $n_{R}$ will have a weak-coupling regime, so long as the one-loop beta function (that depends linearly on $n_{R}$ ) remains asymptotically free. All we need for the next step is that the blocking process in its entirety is taking place in a weak-coupling regime, in both the unrooted and rooted theories.

Next, we argue that the blocked Dirac operator $D_{n}$ and the effective action $S_{\text {eff }}^{(k)}$ (obtained by removing short-distance fermion modes) are local, and that this is true on both the unrooted and rooted ensembles. Functional derivatives of $S_{e f f}^{(k)}$ depend on $H_{k}^{-1}$ and on (derivatives of) $H_{k}$, where the operator $H_{k}=\left[\gamma_{5} \otimes \xi_{5}\right] G_{k}^{-1}$ is hermitian [6]. In view of the discussion below Eq. (3.6), the necessary and sufficient condition for $S_{\text {eff }}^{(k)}$ to be local is that both $H_{k}$ and its inverse be local operators on the $k^{\text {th }}$ lattice scale, in the interacting theory too. Unlike in the free theory, however, we cannot rule out the possibility that $H_{k}$ has no gap in the interacting theory, i.e., that $H_{k}$ may have arbitrarily small eigenvalues. But for the locality of $H_{k}^{-1}$ small eigenvalues are harmless if the eigenmodes are exponentially localized on the corresponding lattice scale. Therefore, a sufficient condition for the locality of $H_{k}^{-1}$, and iteratively for the locality of $D_{k}$ and $H_{k+1}$, is that the mobility edge of $H_{k}$ be $O\left(\alpha_{k}\right)=O\left(1 / a_{k}\right)$. Eigenmodes with eigenvalues above the mobility edge are, by definition, extended. But as long as the extended-modes spectrum does not reach down to zero, the inverse of $H_{k}$ will stay local.

A recent study of the mobility edge of the Wilson operator in the super-critical region reveals a mobility edge remarkably close to the free-theory gap for moderate values of the bare coupling [20]. By interpolation, the mobility edge must be even closer to the free-theory gap in a (really) weakcoupling regime. Intuitively this can be understood as follows. Eigenvalues far below the freetheory gap arise in the presence of "dislocations" in the gauge field. But the pure Yang-Mills action on the $k^{\text {th }}$ lattice strongly suppresses dislocations provided that the running coupling $g_{r}\left(a_{k}\right)$ is very small, in which case the effect of any fermion determinant is subdominant (the large, UV divergent part of the fermion determinant is absorbed into the renormalization of $\left.g_{r}\left(a_{k}\right)\right)$. This works in any renormalizable and asymptotically free (but not necessarily local) theory, in particular for both the ordinary and the rooted staggered ensembles. Having said that, since none of the operators $H_{k}$ has been studied numerically to date, it is clearly important to confirm this part of the argument.

If $D_{n}$ is local then, trivially, $\widetilde{D}_{n}$ and $\Delta_{n}$ are separately local, $c f$. Eq. (3.9). Integrating over all the gauge-fields except $\mathscr{V}^{(n)}$ in Eqs. (3.10) and (3.13), we obtain coarse-lattice theories which are local. In particular, the reweighted theories (3.13) are one-taste theories that belong to the appropriate universality class.

What remains to be established is that the sequence of reweighted (interpolating) theories has

\footnotetext{
${ }^{10}$ See Ref. [12] for a discussion of non-local but renormalizable two-dimensional field theories.
} 
the same $n \rightarrow \infty$ limit as the blocked rooted (unrooted) theory. We now derive this result assuming that, in both the interpolating theories (3.10) and the reweighted theories (3.13), the following scaling relations are valid in an ensemble average sense:

$$
\begin{aligned}
\left\|D_{n}^{-1}\right\| & \lesssim \frac{1}{m_{r}\left(a_{c}\right)} \\
\left\|\Delta_{n}\right\| & \lesssim \frac{a_{f}}{a_{c}^{2}}=\frac{2^{-(n+1)}}{a_{c}}
\end{aligned}
$$

where $m_{r}\left(a_{c}\right)>0$ is the renormalized quark mass. The scaling law (3.15) of the taste-breaking part $\Delta_{n}$ of the Dirac operator neglects logarithmic corrections. It is the anticipated scaling based on the smallest engineering dimension of an irrelevant operator, namely five. ${ }^{11}$

In the rest of the argument we focus on the rooted theory. We compare the rooted and the reweighted theories at the $n^{\text {th }}$ blocking level starting from one-taste operators constructed from the coarse-lattice fields of the reweighted theory, i.e., operators of the form $\mathscr{O}^{(n)}=\mathscr{O}^{(n)}\left(q^{(n)}, \bar{q}^{(n)}, \mathscr{V}^{(n)}\right)$. For such operators, we reconstruct rooted observables, using that un-normalized expectation values satisfy ${ }^{12}$

$$
\begin{aligned}
\left\langle\mathscr{O}^{(n)}\right\rangle_{n}^{\text {root }} & =\left\langle\mathscr{O}^{(n)} \exp \left[\frac{1}{4} \operatorname{tr} \log \left(1+\Delta_{n}\left[\widetilde{D}_{n} \otimes \mathbf{1}\right]^{-1}\right)\right]\right\rangle_{n}^{\text {reweigh }} \\
& =\left\langle\mathscr{O}^{(n)}\right\rangle_{n}^{\text {reweigh }}\left(1+O\left(\varepsilon_{n}^{2}\right)\right)
\end{aligned}
$$

where we have used the taste-tracelessness of $\Delta_{n}$, and where, using Eqs. (3.14) and (3.15),

$$
\varepsilon_{n} \equiv\left\|D_{i n v, n}^{-1}\right\|\left\|\Delta_{n}\right\| \lesssim \frac{a_{f}}{a_{c}^{2} m_{r}\left(a_{c}\right)}=\frac{2^{-(n+1)}}{a_{c} m_{r}\left(a_{c}\right)}
$$

The (anticipated) scaling of $\Delta_{n}$ thus implies that $\varepsilon_{n} \rightarrow 0$ for $n \rightarrow \infty$. The observation here is that, first, after sufficiently many blocking steps the expansion of the logarithm is convergent, and second, that in the limit $n \rightarrow \infty$ the expectation value of $\mathscr{O}^{(n)}$ is the same for the rooted and for the reweighted theories. ${ }^{13}$ This is precisely what is needed for the validity of the rooted theory in the continuum limit! A corollary is that the continuum-limit theory enjoys every exact symmetry that exists in the (rooted) staggered and/or in the reweighted theory.

\subsection{Scaling}

Let us now take a closer look at the scaling laws. A standard perturbative treatment in either the unrooted or the rooted theory would predict all the scaling laws we have used: for the running

\footnotetext{
${ }^{11}$ See Ref. [21] for a first direct study of the scaling of $\Delta_{n}$.

${ }^{12}$ Eq. (3.16) only relates a subset of the observables in the rooted theory - those in the physical subspace — to those of the reweighted theory. If we want to relate all rooted observables, we can (a) leave the reweighted theory as a theory of four rooted, but equivalent, tastes, as was done for the continuum theory in Eq. (1.2), or (b) write the reweighted theory as a theory of four unrooted tastes and three ghost tastes, as in Appendix B of Ref. [12].

${ }^{13}$ Strictly speaking, one should take into account the fact that the valence propagators coming from $\mathscr{O}^{(n)}$ are also slightly different on the two sides of Eq. (3.16): The rooted theory keeps taste-violating terms in these propagators but the reweighted theory does not. The difference is also $O\left(\varepsilon_{n}^{2}\right)$ and thus not important to the argument.
} 
of $g_{r}$ and $m_{r}$, and for the scaling of taste-breaking irrelevant terms. But this falls short of what we need.

Scaling laws for irrelevant operators are usually derived in a Symanzik effective-action context [22], where one imagines integrating out the short-distance fluctuations of all the lattice fields. Here, in contrast, the scaling laws must hold in Eq. (3.13) (or in Eq. (3.10)), where a whole "tower" of gauge fields is still present. How do we know that any scaling law still applies? We have only assumed that the scaling laws (3.14) and (3.15) hold in an ensemble average sense. Furthermore, we have only assumed these scaling laws within the reweighted theories, and these have a local pathintegral representation. We do not rely on the validity of any scaling laws for irrelevant operators directly in the rooted theory.

Operationally, this means that the scaling laws must hold for the expectation values of operators constructed from the coarse-lattice fields of the reweighted theory. Before we can compute any scaling law, we must first set up perturbation theory. Usually lattice perturbation theory begins with the expansion of the link variables as $U_{\mu, x}=\exp \left(\operatorname{igaA}_{\mu, x}\right)$. In a reweighted theory a similar expansion will have to be applied to the tower of gauge fields $\mathscr{U}, \mathscr{V}^{(0)}, \mathscr{V}^{(1)}, \ldots, \mathscr{V}^{(n)}$ simultaneously. With this, the perturbative expansion can in principle be derived directly from Eq. (3.13), because the closed-form expressions for $D_{n}, \widetilde{D}_{n}$ and $S_{\text {eff }}^{(k)}$ as functionals of all the gauge fields are known. One would then proceed to calculate correlation functions with coarse-lattice fields only on the external legs. Because only coarse-lattice gauge fields occur on any external leg, we may imagine that the integrations over $\mathscr{U}, \mathscr{V}^{(0)}, \mathscr{V}^{(1)}, \ldots, \mathscr{V}^{(n-1)}$ are always done before the integrating over $\mathscr{V}^{(n)}$. Since, in addition, all the external momenta are of order $1 / a_{c}$, this brings us closer to a standard RG setup where coarse-lattice observables are computed using a conventional (but complicated) coarse-lattice action (that includes for example multi-fermion interactions). As we explain below, some differences still remain. But first we turn to a conceptual question.

There is a crucial difference between the running of $g_{r}$ and $m_{r}$, and the anticipated scaling of an irrelevant operator. The running of relevant and marginal parameters originates directly from the short-distance divergences of the theory. Because power-counting and renormalizability survive rooting, we have no reason to doubt the validity of rooted perturbation theory for these specific scaling laws. This is especially true given that only general features of the running coupling and mass parameters are needed, but not any details.

In contrast, the anticipated scaling of any irrelevant operator (which does not mix with relevant or marginal operators) implies the vanishing of that operator in the continuum limit. That something should vanish in a certain limit is a more delicate claim. In local theories we have no reason to doubt the perturbative prediction, which amounts to the assumption that no non-perturbative effects interfere with the vanishing of all amplitudes with an insertion of the irrelevant operator. But, the question is, how can we be sure that the non-locality of the rooted theory does not modify the scaling of irrelevant operators in undesirable ways not captured by perturbation theory? Once again, our solution is to rely only on the predictions of perturbation theory in the local reweighted theories. Because the non-locality has been eliminated, we see no reason to trust reweighted perturbation theory any less than perturbation theory for, say, unrooted staggered fermions. This applies in particular to the scaling of (taste breaking) irrelevant operators within the reweighted rooted theory, which, we expect, would give rise to Eq. (3.15).

In the unrooted theory, an analogous analysis starts off with the reweighted unrooted theory, 
i.e., the $t=0$ interpolating theory ( $c f$. Eq. (3.10)), and ends with the uncontroversial conclusion that exact taste symmetry is recovered in the staggered theory in the continuum limit. But, in the unrooted staggered theory we could instead rely directly on unrooted perturbation theory for the scaling of $\Delta_{n}$, because the unrooted theory itself is local.

Within reweighted unrooted perturbation theory, the scaling (3.15) should hold as we go down from $1 / a_{f}$ to $1 / a_{c} \cdot{ }^{14}$ This, in turn, should result from the similar symmetry patterns of the staggered and the reweighted theories, for $n$ large enough. On the staggered side, both taste symmetry and (softly broken) chiral symmetry in the continuum limit are secured by the lattice symmetries. On the reweighted side, we have exact taste symmetry by construction. But if the continuum limit is to come out right, then the reweighted theory should in addition have an approximate chiral symmetry (namely, the additive renormalization of the taste-singlet mass term goes to zero with increasing $n$ ). Indeed, since the $t=0$ and $t=1$ theories in Eq. (3.10) are connected by a convergent expansion, this must be the case.

We can summarize our line of reasoning as follows. First, we claim that the reweighted version of the unrooted staggered theory is local, and that $\Delta_{n}$ scales as expected in that theory. If one accepts the validity of the unrooted staggered theory this claim can be considered "safe." Then, proceeding from the unrooted reweighted theory, which has four tastes and exact $U(4)$ taste symmetry, we can consider the theory in which we take the fourth root of the fermion determinant, and obtain a local one-taste theory in which $\Delta_{n}$ still scales as an irrelevant operator. Of course, the gauge ensemble is different for the reweighted versions of the rooted and unrooted theories. But $\Delta_{n}$ should remain irrelevant as we move from the unrooted reweighted to the rooted reweighted case since perturbation theory should be equally trustworthy for both local theories. Note that this is a claim about reweighted theories only, with no reference to the underlying staggered theory. In the final step, the rooted staggered theory is reconstructed with the help of Eq. (3.16), and the scaling of $\Delta_{n}$ in the rooted reweighted theory ensures that the rooted staggered theory has the desired continuum limit.

Finally, we address a more practical issue. We have argued that QCD is correctly described by the observables of the coarse-lattice (rooted, staggered) theory in the limit of infinitely many blocking steps. Thanks to the pull-back mapping every coarse-lattice observable is at the same time a fine-lattice observable and, as such, it can in principle be computed directly on the (rooted) staggered ensemble. This amounts to using smeared staggered fermion sources constructed in a particular way from all the blocking kernels. The question is, are we allowed to use any other (local) fine-lattice interpolating fields, as is done in practice? Normally, one proves that once renormalization factors have been correctly taken into account, different interpolating fields must give rise to the same physical predictions. But once again we face the difficulty that, because of the non-locality, it may be dangerous to rely on rooted staggered perturbation theory. We believe that, here too, the resolution is to rely on reweighted perturbation theory. By its very construction, the reweighted theory "knows" about fine- and coarse-lattice fields alike, and, therefore, it is capable of comparing coarse-lattice and fine-lattice interpolating fields. We expect that the usual statements about independence of the physical predictions of any particular choice of the interpolating fields will remain true once this comparison is carried out. This can in principle be tested in perturbation theory.

\footnotetext{
${ }^{14}$ Note that this does not amount to a standard blocking process within the reweighted theory.
} 


\section{Staggered chiral perturbation theory}

Here we discuss point 3 of the Introduction, namely the low-energy effective theory for staggered quarks. By definition, such a theory must include discretization effects, in particular tasteviolations, although we expect the theory will go over into continuum chiral perturbation theory $(\chi \mathrm{PT})$ in the $a \rightarrow 0$ limit. When the underlying staggered action is unrooted, finding the effective theory is straightforward $[23,24]$. We call the result "unrooted staggered chiral perturbation theory," or uS $\chi$ PT. On the other hand, the effective theory must be non-trivial in the presence of rooting, since we know from Sec. 2 that non-localities are present, and that their effects show up in the pion sector.

In Ref. [24], it was proposed that the rooting could be taken into account at the chiral level by locating the presence of sea-quark loops in the meson diagrams of uS $\chi \mathrm{PT}$ and multiplying each by $1 / 4$. These loops can be found by quark-flow arguments [25], but for present purposes it is more useful to use the replica trick [26], which was already employed in Sec. 3.3 for weakcoupling perturbation theory. This version of staggered chiral perturbation theory will be referred to as rS $\chi$ PT. We emphasize that the " $r$ " in $\mathrm{rS} \chi \mathrm{PT}$ stands, in the first instance, for "replica." The rules of rS $\chi \mathrm{PT}$, described in more detail below, give a well defined procedure for computing chiral amplitudes. The question is, however, whether $\mathrm{rS} \chi \mathrm{PT}$ is in fact the proper low-energy theory for rooted staggered quarks. We will argue that it is correct; in other other words, we argue that the " $r$ " in $\mathrm{rS} \chi \mathrm{PT}$ also stands for "rooted."

Finding the correct chiral theory is important for several reasons. The discussion in Sec. 2 indicates that the potential problems from rooting show up in the IR, because of the interplay of the physical $\left(m_{\pi}^{G B}\right)$ and unphysical $\left(a \Lambda_{Q C D}^{2}\right)$ IR scales. ${ }^{15}$ Since the chiral theory describes the most IR part of the theory, it provides a laboratory for studying the effects of non-locality and seeing (one hopes) how they go away as $a \rightarrow 0$. Indeed, as mentioned in Sec. 2, rS $\chi$ PT already shows unitarity violations at $a \neq 0[13,14,7]$. Moreover, even if the validity of the rooting procedure were rigorously established at the quark level, the chiral theory would still be crucial for controlling the chiral and continuum extrapolation of simulation results [27]. Since the effects of taste violations are significant at lattice spacings available currently and in the foreseeable future, we cannot do without a chiral theory that takes such effects into account. Finally, the chiral theory provides a non-perturbative handle for relating the unrooted staggered valence sector to the rooted staggered sea sector. In particular, if $\mathrm{rS} \chi \mathrm{PT}$ is indeed correct, it can be used to show that even though the valence sector is unrooted, the theory does not behave as a "mixed" theory in which valence and sea quarks have different lattice actions.

The uS $\chi \mathrm{PT}$ theory for a single unrooted staggered field (i.e., one flavor) is derived through $\mathscr{O}\left(a^{2}\right)$ by Lee and Sharpe [23]. They start by using the staggered symmetries to find the Symanzik action through $\mathscr{O}\left(a^{2}\right)$. Spurions can then be introduced for all terms that violate the taste and chiral symmetries, and the chiral theory follows. The generalization of this procedure to multiple flavors appears in Ref [24]. In the unrooted case, the generalization is straightforward. The proposal to take into account the fourth root, is, in the replica approach, the following:

\footnotetext{
${ }^{15}$ In the RG analysis of Sec. 3, it is plausible that $\varepsilon_{n}$ (Eq. (3.17)) over-estimates the relative size of successive terms in the expansion of the logarithm in Eq. (3.16), and that the relative size is actually $a \Lambda_{Q C D}^{2} / m_{r} \sim a \Lambda_{Q C D}^{3} /\left(m_{\pi}^{G B}\right)^{2}$. This ratio indeed contains the two IR scales $m_{\pi}^{G B}$ and $a \Lambda_{Q C D}^{2}$.
} 
- Replicate the sea-quark degrees of freedom at the chiral level, replacing each by $n_{R}$ identical copies, where $n_{R}$ is a positive integer. All $n_{R}$ copies get the same mass, and, if relevant, the same source terms.

- Calculate order by order in the resulting uS $\chi \mathrm{PT}$, keeping the $n_{R}$ dependence explicit.

- Replace $n_{R}$ by $1 / 4$ at the end.

Note that the dependence on $n_{R}$ is completely determined at any finite order in uS $\chi \mathrm{PT}$, so replacing $n_{R}$ by $1 / 4$ is a well-defined procedure. ${ }^{16}$ As always in a chiral theory, we treat the low-energy constants (LECs) as free parameters for each $n_{R}$; we are just trying to find the dependence of physical quantities on the LECs in the rooted staggered theory.

The argument [7] that $\mathrm{rS} \chi \mathrm{PT}$ is the correct chiral theory for rooted staggered quarks stays completely within the context of chiral theories. As emphasized by Sharpe [12], this is possible because the unfamiliar unphysical features of the rooted theory can be connected to the much more familiar, but still unphysical, features of a partially quenched (and unrooted) theory. While PQ $\chi$ PT, the chiral theory in the partially quenched case [19], is not on as firm a theoretical footing as is ordinary $\chi$ PT for full (unquenched) theories [28], we have a large body of numerical evidence that PQ $\chi$ PT (and the completely quenched version Q $\chi$ PT $[29,25]$ ) are in fact the correct chiral descriptions of the corresponding lattice theories. The evidence comes from Wilson, domain wall, and (for the quenched case) overlap quarks, as well as staggered simulations. Furthermore, PQ $\chi$ PT will be subject to even more stringent numerical tests in the future. So connecting the rooted case to the partially quenched case is a useful step forward.

Reference [7] starts by noting that we know (trivially) how the fourth root works when there are four degenerate flavors $\left(n_{F}=4\right)$. Since the fourth power of the fourth root reproduces the determinant, the rooted $n_{F}=4$ theory is identical to the unrooted $n_{F}=1$ theory. We therefore know the starting chiral theory, namely the uS $\chi \mathrm{PT}$ of Lee and Sharpe. To get to a non-degenerate 4flavor theory, we can expand around the degenerate, massive point; this is where partial quenching is needed. Finally, to get to a theory with $n_{F}<4$ flavors, one quark at a time can be decoupled. Each step in the procedure requires some assumptions, which are plausible but unproven. However, most of the assumptions can be tested numerically, and some already have been tested.

To explain the argument in more detail we need some notation. Let $\left(n_{F}, n_{T}, n_{R}\right)_{L Q C D}$ be the generating functional for a lattice QCD theory with $n_{F}$ flavors, $n_{T}$ tastes, and $n_{R}$ replicas of each flavor; let $\left(n_{F}, n_{T}, n_{R}\right)_{\chi}$ be the generating functional of the corresponding chiral theory. Whenever $n_{R}$ is shown explicitly it is taken to be a positive integer; the end result of the replica trick is indicated by replacing $n_{R}$ with $1 / 4$. When $n_{R}$ is trivially equal to 1 (because the replica trick is not relevant), it is omitted. An unrooted theory is indicated by $n_{T}=4$; while for the rooted theory we put $n_{T}=1$.

Thus $(1,4)_{L Q C D}$ is the theory of a single unrooted staggered field, and $(1,4)_{\chi}$ is the uS $\chi$ PT of Ref. [23]. Similarly, $\left(n_{F}, 4, n_{R}\right)_{\chi}$ is the uS $\chi \mathrm{PT}$ of Ref. [24] with $n_{R} \cdot n_{F}$ sea-quark species. Further, $\left(n_{F}, 1\right)_{L Q C D}$ is the lattice theory of $n_{F}$ rooted staggered fields, and $\left(n_{F}, 1\right)_{\chi}$ is by definition the lowenergy theory generated by integrating out all the higher modes in $\left(n_{F}, 1\right)_{L Q C D}$. As far as we know

\footnotetext{
${ }^{16}$ In Ref. [7], it was claimed that the dependence on $n_{R}$ is strictly polynomial. That is in fact an oversimplification, since we would like to sum the geometric series of hairpin diagrams to all orders, which introduces factors of $n_{R}$ into the masses of flavor-neutral mesons, and hence into the denominators of Feynman integrands. But the key point remains: the functional form of the $n_{R}$ dependence is completely determined at any finite order in uS $\chi \mathrm{PT}$.
} 
so far, $\left(n_{F}, 1\right)_{\chi}$ could be horribly non-local, non-unitary, or otherwise sick. The claim, however, is that $\left(n_{F}, 1\right)_{\chi}$ is in fact $\left(n_{F}, 4, \frac{1}{4}\right)_{\chi}$ to any finite order in chiral perturbation theory, where the latter simply defines what we mean by $\mathrm{rS} \chi \mathrm{PT}$.

The first steps of the argument are taken in the $n_{F}=4$ case. We want to show that

$$
(4,1)_{\chi} \doteq\left(4,4, \frac{1}{4}\right)_{\chi}
$$

where "․" is used to indicate that the two sides are the same functions of the LECs. As mentioned above, we start with a degenerate $n_{F}=4$ theory, with mass matrix $\mathscr{M}=\bar{m} I$, where $I$ is the identity matrix in flavor and taste space. In such as theory, we have

$$
\begin{aligned}
&\left.(4,1)_{L Q C D}\right|_{\mathscr{M}=\bar{m} I}=\left.(1,4)_{L Q C D}\right|_{\bar{m}} \\
&\left.\left.\left.(4,1)_{\chi}\right|_{\mathscr{M}=\bar{m} I} \doteq(1,4)_{\chi}\right|_{\bar{m}} \doteq\left(4,4, \frac{1}{4}\right)_{\chi}\right|_{\mathscr{M}=\bar{m} I}
\end{aligned}
$$

The last equivalence here is manifest order by order in $\mathrm{rS} \chi \mathrm{PT}$, since taking $4 n_{R}$ degenerate flavors and then putting $n_{R}=1 / 4$ gives the same chiral expansion as in a one-flavor theory.

To move away from degenerate limit, we add taste-singlet scalar sources $s^{i j}$ for the sea-quark fields:

$$
\begin{aligned}
\mathscr{L}_{(4,1)} & =\ldots+\bar{m} \bar{\Psi}_{i}(x) \Psi_{i}(x)+\bar{\Psi}_{i}(x) s^{i j}(x) \Psi_{j}(x)+\ldots \\
\mathscr{L}_{\left(4,4, n_{R}\right)} & =\ldots+\bar{m} \bar{\Psi}_{i}^{r}(x) \Psi_{i}^{r}(x)+\bar{\Psi}_{i}^{r}(x) s^{i j}(x) \Psi_{j}^{r}(x)+\ldots
\end{aligned}
$$

with implicit sums over the flavor indices $i, j$ and the replica index $r$. In the $(4,1)_{L Q C D}$ theory the fourth root is taken after the above sources have been included in the determinant.

When $s \neq 0,\left(4,4, \frac{1}{4}\right)_{\chi}$ might not be the right chiral theory, so we define the mismatch by

$$
(4,1 ; s)_{\chi} \doteq\left(4,4, \frac{1}{4} ; s\right)_{\chi}+V[s]
$$

The mismatch $V[s]$ can be expanded around $s=0$, where it is known to vanish. In fact, it can be shown [7] that all derivatives of $(4,1 ; s)_{\chi}$ and $\left(4,4, \frac{1}{4} ; s\right)_{\chi}$ with respect to the sources are equal:

$$
\left.\left.\prod_{n} \frac{\partial}{\partial s^{i_{n} j_{n}}\left(x_{n}\right)}(4,1 ; s)_{\chi}\right|_{s=0} \doteq \prod_{n} \frac{\partial}{\partial s^{i_{n} j_{n}}\left(x_{n}\right)}\left(4,4, \frac{1}{4} ; s\right)_{\chi}\right|_{s=0}
$$

which implies

$$
\left.\prod_{n}\left(\frac{\partial}{\partial s^{i_{n} j_{n}}\left(x_{n}\right)} V[s]\right)\right|_{s=0}=0 .
$$

Equation (4.6) is proved by first relating sea-quark correlation functions in each theory to partially quenched valence-quark correlation functions, where the valence quarks have the same lattice action as the sea quarks. This then allows us to set the sea-quark source $s=0$, where the equivalence of the two chiral theories is known. The proof requires the existence of standard PQ $\chi \mathrm{PT}$ for unrooted theories, namely for $(1,4)_{\chi}$ and $\left(4,4, n_{R}\right)_{\chi}$. We note that partially quenched theories are crucially needed because the derivatives with respect to sea-quark sources in a rooted theory result in different factors of $1 / 4$ in different contractions, which can never happen in the sea-quark sector of an unrooted theory. 
Thus all derivatives of $V[s]$ vanish at $s=0$. If $V[s]$ is analytic in $s-$ up to possible isolated singularities - it must vanish everywhere, and $(4,1 ; s)_{\chi} \doteq\left(4,4, \frac{1}{4} ; s\right)_{\chi}$. Since an arbitrary fourflavor mass matrix can be obtained by a suitable choice of $s$, Eq. (4.1) is verified. In other words, rS $\chi$ PT (i.e., $\left(4,4, \frac{1}{4}\right)_{\chi}$ ) is the right chiral theory in the four-flavor case.

The assumption of analyticity could fail in a two ways. First of all, there could be a phase transition when the masses are some finite distance away from the degenerate point. There is some evidence from simulations [27] that this does not occur, although it is still possible that a phase transition lurks beyond the range of parameters (masses, lattice spacings) that have been investigated to date. Analyticity could also fail if there were an essential singularity right at $s=0$. This is hard to rule out a priori, though it seems unlikely, since we are expanding around a massive theory with no obvious IR divergences. At the end of this section, we discuss work in progress on deriving $\mathrm{rS} \chi \mathrm{PT}$ directly from the lattice theory, using the methods of Sec. 3. If successful, this work would (among other advantages) reduce these analyticity concerns.

To move from $n_{F}=4$ to $n_{F}=3$, we take one quark mass large. Call it the "charm" quark, with mass $m_{c}$. We first choose $m_{c}$ as large as possible without leaving the region where chiral perturbation theory applies. This point is taken, nominally, as $m_{c} \sim 2 m_{s}^{\text {phys }}$, where $m_{s}^{\text {phys }}$ is the physical strange quark mass. For a clean separation of scales, we can temporarily take the other three masses much smaller than $m_{s}^{\text {phys }}$.

We then integrate out $m_{c}$ from $\left(4,4, \frac{1}{4}\right)_{\chi}$. Since this is a perturbative process (order by order in $\mathrm{rS} \chi \mathrm{PT})$, there is little doubt that the resulting chiral theory is $\left(3,4, \frac{1}{4}\right)_{\chi}$, just as continuum $S U(2)_{L} \times$ $S U(2)_{R} \chi \mathrm{PT}$ results from integrating out the strange quark from the $S U(3)_{L} \times S U(3)_{R}$ theory [30]. Nevertheless, an explicit check of this step is in progress [31].

Since, by the previous steps in the argument, $\left(4,4, \frac{1}{4}\right)_{\chi}$ describes the long-distance physics of the $n_{F}=4$ theory, $\left(3,4, \frac{1}{4}\right)_{\chi}$ describes that physics when $m_{c} \sim 2 m_{s}^{\text {phys }}$. We now assume that this decoupling of the charm quark from the long-distance physics remains true (up to the usual renormalizations of relevant and marginal operators) as $m_{c}$ increases still further, until $m_{c} \gg 1 / a$. At that point, $m_{c}$ is much larger than all the eigenvalues of the Dirac operator, and charm must decouple from the lattice theory, leaving $\left(3,4, \frac{1}{4}\right)_{L Q C D}$. Under our assumptions, we then have

$$
(3,1)_{\chi} \doteq\left(3,4, \frac{1}{4}\right)_{\chi}
$$

So $\mathrm{rS} \chi \mathrm{PT}$ is the correct chiral theory for three rooted staggered flavors. We can then repeat the steps to argue $(2,1)_{\chi} \doteq\left(2,4, \frac{1}{4}\right)_{\chi}$, and $(1,1)_{\chi} \doteq\left(1,4, \frac{1}{4}\right)_{\chi}$.

These results immediately suggest an apparent paradox, which is seen most clearly in the $n_{F}=1$ case. The theory with one flavor should have only a heavy pseudoscalar, which we call the $\eta^{\prime}$, and no light pseudo-Goldstone bosons. Yet the theory with one rooted staggered flavor contains light pions, whose masses vanish in the continuum chiral limit, as well as one heavy meson, the taste-singlet $\eta_{I}^{\prime}$. There are different weightings of the contributions of such particles in the chiral theories of the rooted and unrooted cases, but otherwise $\mathrm{rS} \chi \mathrm{PT}$ and $\mathrm{uS} \chi \mathrm{PT}$ are similar.

We first note that the contributions of the unwanted particles must disappear from physical correlation functions in the continuum limit, either by decoupling, or by canceling against each other, or both. This follows from the argument around Eq. (1.2): In the continuum limit taste symmetry is exact, and the physical sector (defined here as the correlation functions generated by taste-singlet sources) is exactly equivalent to a continuum theory of a single flavor. What rS $\chi \mathrm{PT}$ 
adds to the discussion is that it allows us to see exactly how the decouplings and cancellations take place as the continuum limit is approached.

An rS $\chi$ PT calculation of the $n_{F}=1$ taste-singlet scalar-scalar correlator has been performed to one loop $[14,7]$. There are two kinds of unphysical contributions that appear at $a \neq 0$. Terms coming from the taste-violating hairpins [24] involve taste-vector and taste-axial-vector pions, and are proportional to explicit powers of $a^{2}$ - they simply vanish in the continuum limit. There are also contributions from the physical, taste-singlet hairpins due to the anomaly, as well as from connected (non-hairpin) meson correlators. These generate a two- $\eta_{I}^{\prime}$ intermediate state with the physical weight, but also two-pion states. When we let $n_{R} \rightarrow 1 / 4$, the latter states have relative weights: $1,4,6,4,-15$, for the taste-pseudoscalar (Goldstone), axial, tensor, vector, and singlet pions, respectively. ${ }^{17}$ The existence of the term with negative weight here is a clear indication of unitarity violations. At $a \neq 0$, these pions have different masses due to taste-symmetry violation and have a non-zero contribution to the correlation function. The sum of the weights is 0 , however, so the contributions cancel in the continuum limit when all the pions become degenerate.

If, as argued, $\mathrm{rS} \chi \mathrm{PT}$ is the correct chiral theory for rooted staggered quarks, then there are some important consequences. When $a \rightarrow 0,\left(n_{F}, 4, n_{R}\right)_{\chi}$ becomes ordinary $\chi \mathrm{PT}$ for $4 n_{F} \cdot n_{R}$ quark species. Therefore, taking $n_{R} \rightarrow 1 / 4$ order by order produces standard, continuum $\chi \mathrm{PT}$ for $n_{F}$ flavors in the physical sector. ${ }^{18}$ This then implies that the lowest-energy regime of $n_{F}$-flavor lattice QCD with rooted staggered quarks becomes indistinguishable in structure in the continuum limit from ordinary $n_{F}$-flavor QCD. In this limit, there will therefore be no unitarity or locality violations in the physical sector of the chiral theory. Of course, assuming the arguments of Sec. 3 go through, this had to work, since the continuum limit of the rooted staggered lattice theory is true QCD.

Another use of $\mathrm{rS} \chi \mathrm{PT}$ is more technical. It has been suggested (e.g., in Ref [32]) that the theory of rooted staggered sea quarks and (necessarily unrooted) staggered valence quarks is a "mixed" theory, which behaves like it has different lattice actions for the valence and sea sectors. If that were the case, it would be rather unpleasant: Among other features, mixed theories have different renormalizations of sea and valence quark masses, and even if the quark masses are tuned to make the sea-sea and valence-valence mesons degenerate, the valence-sea mesons will be split from the others by discretization errors [33].

In weak-coupling perturbation theory, it is easy to see with the replica trick that the rooted staggered theory does not behave like a mixed theory: the renormalizations in the sea and valence sectors are the same $[7,5]$. Furthermore, $\mathrm{rS} \chi \mathrm{PT}$ allows us to argue that the theory is not mixed at the chiral level either. The point is that $\left(n_{F}, 4, \frac{1}{4}\right)_{\chi}$ is obtained order by order from $\left(n_{F}, 4, n_{R}\right)_{\chi}$. The latter theory in turn has lattice symmetries (broken only by mass terms) that interchange valence and sea quarks. These symmetries imply that the theory of rooted staggered sea quarks and staggered valence quarks behaves like a partially quenched theory, not a mixed theory. One can give different masses to the sea and valence quarks, as usual in a partially quenched situation. However, if one chooses to give valence and sea quarks the same masses, then the symmetries forbid, for example, the splitting of valence-sea from valence-valence or sea-sea mesons.

Finally, we briefly describe our work in progress [34], in which we attempt to derive rS $\chi \mathrm{PT}$

\footnotetext{
${ }^{17}$ See Sec. VI of Ref. [7] for a detailed discussion of how these weights arise in rS $\chi$ PT.

${ }^{18} \mathrm{We}$ are considering here the case of positive quark masses only. For a discussion of the issues involved with negative masses, see Refs. [3, 4].
} 
directly from the rooted staggered lattice theory. The immediate problem in such a derivation is that a straightforward replica trick fails at the non-perturbative QCD level because we have no control over the functional dependence of the theory on $n_{R}$. In weak-coupling QCD perturbation theory the dependence on $n_{R}$ arises only from the counting of sea quark loops, and thus is manifest order by order. But once we move beyond perturbation theory, the $n_{R}$ dependence is not known a priori, and a unique analytic continuation from integer $n_{R}$ to $n_{R}=1 / 4$ is not possible.

This problem with the replica trick at the QCD level is to be contrasted with the trick at the chiral level: rS $\chi$ PT makes sense because the dependence on $n_{R}$ is known when we calculate order by order in chiral perturbation theory. The unknown $n_{R}$ dependence of the QCD level is hidden in the $n_{R}$ dependence of the LECs of the corresponding chiral theory. Since the goal in the chiral theory is simply to calculate physical quantities as functions of the LECs, which are treated as independent variables, we can (must!) ignore the hidden $n_{R}$ dependence of the LECs in rS $\chi \mathrm{PT}$.

Our tentative solution to this problem is to use the reweighted theory of Sec. 3 as an intermediate step. Since this is a local theory, finding the corresponding chiral theory is straightforward. We then need the replica trick only to move from the reweighted theory to the rooted staggered case. The latter can be obtained by a convergent Taylor expansion (in the parameter $t$ introduced after Eq. (3.9)) around the former. This makes it possible to control the $n_{R}$ dependence at the QCD level, which should allow us to establish an unambiguous connection between the QCD and chiral levels, and thereby to derive rS $\chi$ PT directly. It should be noted, however, that such a derivation would still rely on the existence of PQ $\chi \mathrm{PT}$ for standard (local) partially quenched theories.

\section{Acknowledgments}

We thank Steve Sharpe and Andreas Kronfeld for useful discussions. All three authors thank the Institute for Nuclear Theory at the University of Washington, Seattle, for hospitality; CB also thanks the Centre de Physique Théorique, Marseille. MG was supported in part by the Generalitat de Catalunya under program PIV1-2005. CB and MG are supported in part by the US Department of Energy, and YS is supported by the Israel Science Foundation under grant 222/02-1.

\section{References}

[1] J. B. Kogut and L. Susskind, Phys. Rev. D 11, 395 (1975).

[2] N. Kawamoto and J. Smit, Nucl. Phys. B 192, 100 (1981).

[3] C. Bernard, M. Golterman, Y. Shamir and S. R. Sharpe, hep-lat/0603027.

[4] S. Dürr and C. Hoelbling, Phys. Rev. D 74, 014513 (2006) [hep-lat/0604005].

[5] Y. Shamir, hep-lat/0607007.

[6] C. Bernard, M. Golterman and Y. Shamir, Phys. Rev. D 73, 114511 (2006) [hep-lat/0604017].

[7] C. Bernard, Phys. Rev. D 73, 114503 (2006) [hep-lat/0603011].

[8] M. Creutz, hep-lat/0603020.

[9] M. Golterman and J. Smit, Nucl. Phys. B 245, 61 (1984); M. Golterman, Nucl. Phys. B 273, 663 (1986). 
[10] Y. Shamir, Phys. Rev. D 71, 034509 (2005) [hep-lat/0412014].

[11] F. Gliozzi, Nucl. Phys. B 204, 419 (1982); A. Duncan, R. Roskies and H. Vaidya, Phys. Lett. B 114, 439 (1982). H. Kluberg-Stern, A. Morel, O. Napoly and B. Petersson, Nucl. Phys. B 220, 447 (1983).

[12] S. Sharpe, contribution to these proceedings.

[13] S. Prelovsek, [hep-lat/0510080].

[14] C. Bernard, C. DeTar, Z. Fu, and S. Prelovsek, contribution to these proceedings, and in preparation.

[15] J. Smit and J. C. Vink, Nucl. Phys. B 286, 485 (1987); S. Dürr and C. Hoelbling, Phys. Rev. D 69, 034503 (2004) [hep-lat/0311002] and Phys. Rev. D 71, 054501 (2005) [hep-lat/0411022]; C. Bernard, Phys. Rev. D 71, 094020 (2005) [hep-lat/0412030].

[16] E. Follana, A. Hart and C. T. H. Davies, Phys. Rev. Lett. 93, 241601 (2004) [hep-lat/0406010]; S. Dürr, C. Hoelbling and U. Wenger, Phys. Rev. D 70, 094502 (2004) [hep-lat/0406027];

[17] M. Golterman, Y. Shamir and B. Svetitsky, contribution to these proceedings.

[18] T. Balaban, M. O’Carroll and R. Schor, Commun. Math. Phys. 122, 233 (1989); Lett. Math. Phys. 17, 209 (1989).

[19] C. W. Bernard and M. F. L. Golterman, Phys. Rev. D 49, 486 (1994) [hep-lat/9306005].

[20] M. Golterman and Y. Shamir, Phys. Rev. D 68, 074501 (2003) [hep-lat/0306002]; M. Golterman, Y. Shamir and B. Svetitsky, Phys. Rev. D 71, 071502 (2005) [hep-lat/0407021]

[21] C. Bernard et al., PoS LAT2005, 114 (2006) [hep-lat/0509176].

[22] S. R. Sharpe, Nucl. Phys. Proc. Suppl. 34, 403 (1994) [hep-lat/9312009]; TASI 1994 lectures, hep-ph/9412243; A. Patel and S. R. Sharpe, Nucl. Phys. B 395, 701 (1993) [hep-lat/9210039]; Nucl. Phys. B 417, 307 (1994) [hep-lat/9310004]; Y.-B. Luo, Phys. Rev. D 55, 353 (1997) [hep-lat/9604025].

[23] W.-J. Lee and S. R. Sharpe, Phys. Rev. D 60, 114503 (1999) [hep-lat/9905023].

[24] C. Aubin and C. Bernard, Phys. Rev. D 68, 034014 (2003) [hep-lat/0304014] and Phys. Rev. D 68, 074011 (2003) [hep-lat/0306026]; C. Bernard, Phys. Rev. D 65 (2002) 054031 [hep-lat/0111051].

[25] S. R. Sharpe, Phys Rev. D 46, 3146 (1992) [hep-lat/9205020].

[26] P. H. Damgaard and K. Splittorff, Phys. Rev. D 62, 054509 (2000) [hep-lat/0003017].

[27] C. Aubin et al. [MILC Collaboration], Phys. Rev. D 70, 114501 (2004) [hep-lat/0407028]; C. Aubin et al. [HPQCD, MILC, and UKQCD Collaborations], Phys. Rev. D 70, 031504(R) (2004)

[hep-lat/0405022]; C. Aubin et al. [Fermilab Lattice, MILC, and HPQCD Collaborations], Phys. Rev. Lett. 95, 122002 (2005) [hep-lat/0506030].

[28] S. Weinberg, Physica A 96, 327 (1979).

[29] C. Bernard and M. Golterman, Phys. Rev. D 46, 853 (1992) [hep-lat/9204007].

[30] J. Gasser and H. Leutwyler, Nucl. Phys. B 250, 465 (1985).

[31] C. Bernard and X. Du, work in progress.

[32] A. D. Kennedy, Nucl. Phys. Proc. Suppl. 140, 190 (2005) [hep-lat/0409167].

[33] O. Bär, G. Rupak and N. Shoresh, Phys. Rev. D 67, 114505 (2003) [hep-lat/0210050] and Phys. Rev. D 70, 034508 (2004) [hep-lat/0306021]; O. Bar, C. Bernard, G. Rupak and N. Shoresh, Phys. Rev. D 72, 054502 (2005) [hep-lat/0503009].

[34] C. Bernard, M. Golterman, and Y. Shamir, in preparation. 